\title{
The shape of the cup influences aroma, taste, and hedonic judgements of specialty coffee
}

\author{
Fabiana M. Carvalho ${ }^{1 *} \&$ Charles Spence ${ }^{2}$ \\ ${ }^{1}$ Department of Philosophy, University of Sao Paulo (USP), Sao Paulo, Brazil \\ ${ }^{2}$ Crossmodal Research Laboratory, Department of Experimental Psychology, University of Oxford, Oxford, UK \\ * Correspondence: Fabiana Carvalho, University of Sao Paulo, FFLCH, Department of \\ Philosophy, Av. Prof. Luciano Gualberto, 315 - Cidade Universitária, CEP 05508900 - São \\ Paulo - Brasil; bibimesq@gmail.com
}




\begin{abstract}
The drinking experience depends on the multisensory integration of attributes of the drink itself as well as the characteristics of the drinking vessel, not to mention the environment in which the drink happens to be tasted. The receptacles from which we drink have been shown to affect the perception of the sensory and hedonic attributes of various different beverages (especially in the world of wine). The present study was designed to investigate whether the shape of the cup would also influence amateur and/or expert consumers' perception of aroma, taste, and hedonic evaluation of specialty coffee. A large-sample experiment (involving 276 participants) was conducted in a specialty coffee event in Brazil. The participants were divided into three testing groups according to the shape of the cup in which the coffee was served (tulip, open, or split). Tasters evaluated their experience of the aroma, sweetness, acidity, and liking of the coffee. Multivariate analysis of variance was conducted in order to assess the effect of cup shape on sensory and hedonic ratings, and whether expertise modulated these ratings. Both amateurs and experts judged: (1) the aroma to be significantly stronger in the tulip cup, and (2) the sweetness and acidity to be significantly more intense in the split cup. Interestingly, the split cup received the lowest liking scores from the amateurs, but not from the experts. Taken together, these results demonstrate for the first time that the shape of the cup significantly affects the perception of the sensory attributes of specialty coffee, for both amateur and expert consumers. The implications of these results for the design of coffee cups that convey some functional and/or perceptual benefit as well as possible directions for future research are discussed.
\end{abstract}

KEYWORDS: SPECIALTY COFFEE; DRINKING VESSEL; MULTISENSORY; SHAPE. 


\section{Introduction}

The conscious perception of flavour - as a unified sensory and hedonic experience - could be seen as the outcome of perhaps the most complex integration of multisensory and affective signalling by the brain (e.g., Okamoto \& Dan, 2013). The multisensory experience of flavour goes beyond the mere combination of taste, retronasal olfaction, and oralsomatosensory/trigeminal signals that are subjectively localized to the mouth. It involves (or is modulated by) the integration of cues coming from all of our senses, which includes what we see, hear, touch, and smell orthonasally (e.g., Spence, 2015) - the latter helping to set our sensory and hedonic taste/flavour expectations (Spence \& Piqueras-Fiszman, 2014; Verhagen \& Engelen, 2006). However, not only do the physicochemical properties of the food or drink itself convey information that is relevant to the flavour construct, in addition to these productintrinsic cues (e.g., colour, shape, and texture of what is actually consumed), extrinsic cues, such as tableware and packaging, have also been shown to exert an influence on our perception of flavour and hedonic judgements (Spence et al., 2012; Spence \& PiquerasFiszman, 2014).

A growing body of empirical research now shows that multisensory information can be used to improve the design of products. Concerning the influence of shape, it has frequently been demonstrated that the shape of the product itself, the shape of a product's container (e.g., serving utensil, packaging, logotype), and even any shapes that happen to be near to the product can affect many aspects of consumer behaviour (Gal et al., 2007; Lynch \& Zauberman, 2007). This also applies to the shape of drinking receptacles. Our drinking experience is necessarily tied to the receptacle from which a drink is consumed, be it, for instance, a glass, a cup, a mug, or a bottle. In particular, converging evidence now demonstrates strong association between the shape properties of the drinking receptacle and the experience of taste (or flavour) (Spence \& Van Doorn, 2017; Spence \& Wan, 2015; Van Rompay et al., 2017). Compared to all other drinking receptacles, the wine glass has received far more empirical investigation than any other class of vessel/receptacle. Over the last few decades, an extensive body of research has assessed the influence of the shape of the wine glass on the perception of aroma and flavour in both experts and social drinkers. Serving the same wine in differently-shaped glassware has been shown to exert a significant influence on both sensory and hedonic ratings (Cliff, 2001; Hummel et al., 2003; see Spence, 2011, for a 
review). In fact, the claim that particular types of wine (or even varietals) should be matched by specific glass shapes has boosted the development of what is a highly-profitable industry.

The results of the extensive body of research in wine glassware area suggest that, whenever a person - amateur or expert - physically interacts with the glass when evaluating the sensory properties of the wine, then the shape of the glass will affect their ratings. Bulbous glasses increase the aroma and taste ratings of the wine, having a more robust impact on aroma judgements and a less consistent effect on taste (see Spence \& Wan, 2015, for a review). Recently, researchers have begun to explore the impact of glass shape on flavour attributes and hedonic judgements in beer (Mirabito et al., 2017) and other carbonated beverages (Cavazzana et al., 2017). For instance, the Australian pale ale served to participants in Mirabito et al.'s recent study was rated as being fruitier and more intense when served in a curved-sided transparent glass than in a straight-sided receptacle. Meanwhile, the cola in Cavazzana et al.'s study was rated as more pleasant and more intense when tasted from a typical curved-sided cola glass than when tasted from a water glass or plastic bottle instead. ${ }^{1}$ That said, despite the frequently reported correspondence effect between round shapes and sweet taste, one recent study failed to demonstrate the association between a round cup shape and sweet taste (butter milk drink), but a correspondence was found between the angular cup and bitter taste (mate-based soft drink) (Machiels, 2018). These studies raise the question of when consumers use shapes as cues for tastes, that is, when crossmodal effects should be expected.

The correspondence between the shape of the receptacle and flavour attributes in specialty coffee remains essentially unknown. Specialty coffee, as defined by Donnet et al. (2007), "is the revitalization of the art of cultivating, roasting, preparing and enjoying a beverage of superior aroma and flavour." 2 Specialty coffees come from the most exceptional quality beans harvested from the best of the world's Coffea arabica crops, roasted and brewed to

\footnotetext{
1 It should, perhaps, also be noted here that the weight of the drinking receptacles were not matched in this study, and the differences in weight may also have contributed to the differences of assessed ratings (see Gatti et al., 2016; Kampfer et al., 2017).

${ }^{2}$ According to SCA, the term was first coined by Erna Knutsen in 1978, stating in essence that specialty coffees are coffees made from coffee beans grown in special geographic microclimates with unique flavour profiles (Rhinehart, 2009).
} 
enhance their flavour potential. The sensory attributes of specialty coffee are evaluated by Specialty Coffee Association (SCA) cupping certified judges (or Q graders), according to the international Q Coffee System methodology (Lingle \& Menon, 2017). ${ }^{3}$ Specialty coffee represents a growing market segment in an otherwise declining industry. In the U.S., of all the cups of coffee consumed in $2017,41 \%$ were non-specialty versus $59 \%$ specialty (the latter figure was just $40 \%$ in 2010 , when NCA started tracking specialty coffee consumption; SCA, 2017a). In Western Europe, since 2010, the number of coffee focused shops increased by $50 \%$, while the number of cafes (which sell food, coffee, and a variety of other beverages) has declined by $8 \%$ (SCA, 2017b). This indicates the continued desire of the consumer not only for better tasting coffee but also his/her interest for the variety of different coffee types and brewing methods, as well as coffee-related products offered by those establishments focused on coffee. Nevertheless, these specialty coffee shops (and baristas alike) should be thinking beyond just beans and preparation methods - that is, they should be prepared to offer more than just the coffee itself. Drinking specialty coffee can potentially be a truly engaging multisensory experience, one which includes the cup in which the coffee is served as an essential element (Bury, 2014).

Just like wine, specialty coffee is undoubtedly a chemically/perceptually very complex beverage (Spence \& Wang, submitted). Wine has been estimated to contain 600-800 volatile aromatic compounds (Rapp, 1990), whilst around 1,000 volatiles have been reported in coffee (Yeretzian, 2017) ${ }^{4}$. Additionally, by being agricultural products, the terroir of the region in which a certain coffee or wine varietal have grown directly influences the primary sensory characteristics (Barbosa et al., 2012; Styger et al., 2011). Both are processed after harvesting which introduce secondary characteristics to the final sensory profile. Thus, the

\footnotetext{
${ }^{3}$ In this evaluation, grades on a scale of zero to ten are awarded for the attributes of fragrance/aroma, sweetness, acidity, body, flavour, balance, and aftertaste. A 100-point scale is then used to summarize the flavour and aesthetic qualities of a brewed coffee. It is important to mention that dominant bitterness and astringency are negative attributes, and classified as primary defects. Specialty coffees are those coffees with a cupping score of 80 or more points and with no primary defects.

${ }^{4}$ There is no one-to-one correspondence between chemical complexity and perceived complexity (see Spence \& Wang, submitted), with only 30-40 of those volatiles likely actually contributing to the orthonasal aroma/retronasal flavour (see Benzi, 2008). Furthermore, the human psychophysics suggests that people are only able to identify a maximum of 2-4 odours in odour or odour-taste mixtures, even if they are entirely familiar with all of the odours when presented in isolation (Laing \& Francis, 1989; Laing et al., 2002).
} 
combination of varietals, terroir, and post harvesting processing methods creates a huge variation of distinctive characteristics in terms of their body, aroma, and flavour complexity. During the last two decades, the so-called "third-wave" phenomenon has introduced coffee into the general trend of enjoyment of fine foods and drinks. The specialty coffee industry is increasingly becoming the new wine of the fine beverage market, with consumers willing to pay to appreciate coffee for its origin and individual characteristics, and with record prices paid for "Special Reserve Coffees" (Teuber \& Herrmann, 2012).

In addition to the description of the origin and sensory attributes of the product, the marketing strategies for specialty coffee could also be directed towards enhancing the product's appeal to consumers by means of the drinking receptacle used which might provide a functional benefit. Given the empirical evidence, it is plausible to expect that the shape of the coffee cup would exert an influence on tasters' ratings in much the same way as has been demonstrated for wine. On the other hand, the fact that specialty coffee is served at a much higher temperature than wine might well mean that more of the volatiles escape from the headspace over the liquid in the drinking vessel. Clearly, empirical investigation is needed given the surprising lack of published research on this topic, surprising especially given that coffee is one of the world's most widely consumed hot beverages (Xu, 2003).

The present study was designed to investigate whether different shapes of coffee cups produce differences in perceptual evaluations (ratings of aroma and flavour attributes) as well as hedonic judgement of a specialty coffee. The cups selected for testing are already wellknown accessories in the specialty coffee market, and have been advertised as "a unique coffee concept which enhances the coffee drinking experience" 5 . Despite having become increasingly trendy amongst specialty coffee consumers and experts, we are aware of no empirical assessment on whether their shapes actually affect the coffee drinking experience has been conducted yet. Furthermore, these coffee cups appear suitable for testing since they come in shapes - bulbous, open, and indented waist - that are common in the beverage market. Both amateurs and professional consumers were tested in a large-sampling study during a specialty coffee event held in Brazil.

\section{Material and Methods}

\section{1 - Participants}

\footnotetext{
${ }^{5}$ Figgjo Norway website at https://www.figgjo.com/news-figgjo-oslo
} 
Two hundred and eighty-seven participants took part in the study after giving their informed consent. The study was approved by the Research Ethics Committee of the School of Psychology, University of São Paulo. After data quality control, the data from 276 participants were included in the final analysis (see Table 1). Both specialty coffee professionals and amateurs were assessed. The professionals included producers, graders, traders, roasters, and baristas who had been working with specialty coffee for at least a year. No specific knowledge of specialty coffee was required from amateurs, and a questionnaire was used to assess each participant's familiarity and consumption frequency. Amateurs were included in the study only if they had been consuming specialty coffee for at least a year. None of the participants reported having a cold or any other impairment of their sense of smell or taste at the time of the study.

Table 1: Participant demographics.

\begin{tabular}{|c|c|c|c|c|c|c|c|c|c|}
\hline & & & & & \multicolumn{5}{|c|}{ Profession $^{(1)}$} \\
\hline & Male & Female & $\begin{array}{c}\text { Age } \\
(M \pm S D) ; \\
\text { range(years) }\end{array}$ & $\begin{array}{l}\text { Coffee activity } \\
\qquad(M \pm S D) ; \\
\text { range(years) }\end{array}$ & $\mathbf{P}$ & $\mathbf{G}$ & $\mathbf{T}$ & $\mathbf{R}$ & B \\
\hline Professional & 85 & 66 & $35.97 \pm 10.33 ; 19-60$ & $4.16 \pm 2.62 ; 1-13$ & 28 & 36 & 24 & 25 & 38 \\
\hline Amateur & 60 & 65 & $36.01 \pm 10.47 ; 18-64$ & $3.08 \pm 1.85 ; 1-8$ & - & - & - & - & - \\
\hline Total & 145 & 131 & - & - & - & - & - & - & - \\
\hline
\end{tabular}

\section{2 - Stimulus}

Coffee: A single origin Arabica coffee was used in the study. The green coffee beans came from the micro-lot of a production plot (1,020m of altitude) of Recreio Estate Coffee, located in São Sebastião da Grama, on the border between the states of São Paulo and Minas Gerais, Brazil. The cultivar was Yellow Bourbon, and the post-harvest processing method used was a semidry method - the so-called honey or pulped natural process (Schwan et al., 2012). The coffee was roasted in a Probatino roaster (Probat Inc., Germany) at the Coffee Industry Syndicate of São Paulo State (Sindicafé) according to the Specialty Coffee Association protocol (SCA, 2015) in order to achieve a light-medium roast profile (60 to 55 Agtron units - Agtron, Inc., Reno, NV). The coffee sample was graded by SCA-certified sensory analysts (Q-graders) and was chosen based on its medium scores in intensity scales for aroma, acidity, sweetness, and body, as well as its overall score of 86 points. Regarding overall 
aroma/flavour characteristics, the coffee was described as having strong nuances of sweetness (molasses and brown sugar), prominent notes of orange, and clean citrus acidity.

The coffee beverage served to the participants during the assessment was a filter (pour-over) coffee prepared using the Hario V60 Kit (Hario V60; Tokyo, Japan) at a concentration of $77 \mathrm{~g} \cdot \mathrm{L}^{-1}$ obtained by pouring hot mineral water $\left(92^{\circ} \mathrm{C}\right)$ over the roasted and ground coffee. The mean temperature of the coffee served to the participants was $58.2^{\circ} \mathrm{C}(S D=1.8)$.

Cups: The participants were divided into three testing groups according to the shape of the cup from which the coffee was sampled. The cups used were the Oslo cup series from Figgjo (Figgjo, Oslo, Norway) developed by Tim Wendelboe (Oslo, Norway). The cups were chosen because 'shape' (tulip, open, and split - Figure 1) was the only factor that varied between them - i.e., all of the cups were made of the same material (ceramic), and had the same texture (smooth), colour (white), height $(6.5 \mathrm{~cm})$, and virtually the same weight $[$ Mean $(\mathrm{g}) \pm$ SD for tulip (173.0 \pm 1.9$)$, open (176.5 \pm 1.9$)$, and split (175.2 \pm 1.7$)]$.
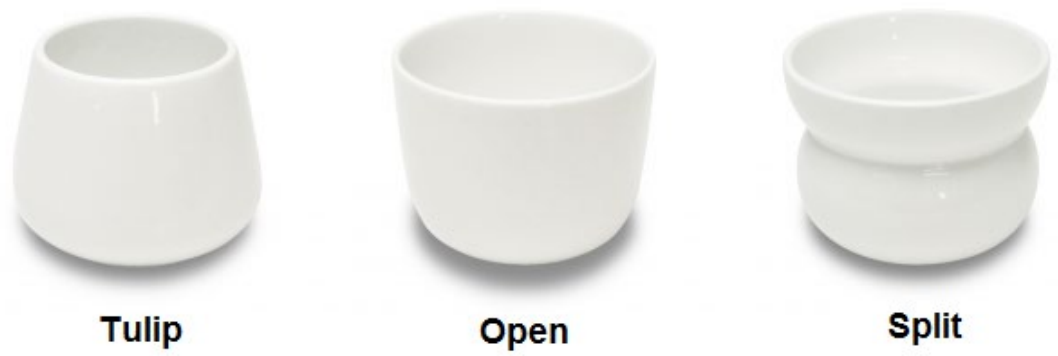

Figure 1: The three different shapes of coffee cups used in the experiment. Their approximate opening and bottom dimensions (in cm) are: Tulip $(6.0 \mathrm{~cm}, 8.5 \mathrm{~cm})$; Open $(8 \mathrm{~cm}, 7 \mathrm{~cm})$; Split $(8 \mathrm{~cm}, 7.5 \mathrm{~cm})$.

\section{3 - Design and procedure}

The experiment was conducted during the $5^{\text {th }}$ International Coffee Week, the largest specialty coffee event held in Brazil. The majority of the participants were recruited primarily through the event website in which they could fill in a short questionnaire and pre-book a testing slot. Those participants who became interested in the study during the event itself were fit into the testing slots where available. Before the start of the study, all of the participants were informed that they would taste and evaluate one sample of specialty coffee. The participants were led, in two groups of six, into a quiet, well-lit air-conditioned testing room. Two 
rectangular tables were placed at the centre of the room, approximately three metres from each other. Each group of six participants was then seated around one of these two tables, with one-metre spacing between adjacent tasters. Each group (i.e., each table) received the coffee sample in the same cup shape. A between-participants experimental design was used. The participants were assigned to each testing condition in order to get a final sampling design that was as balanced as possible.

A sheet containing the rating scales, a pen, and a glass of water were placed in front of each participant's place prior to their arrival at the testing room. At the start of each session, the group of participants received a four-minute briefing to ensure that they were all given the same instructions prior to the experiment. During this time, the coffee was prepared by a professional barista at a bench top in the testing room, but out of sight of the participants. Each participant then received one cup containing approximately $60 \mathrm{~mL}$ of filter coffee. The participants were instructed to evaluate the aroma before tasting the coffee using the aroma rating scale. Next, they moved on to tasting and rating the coffee's temperature, followed by its sweetness and acidity (with the order counterbalanced across participants), and finally how much they liked it. Ratings were performed using a $10-\mathrm{cm}$ visual analog scale anchored at 0 ('not at all') and 10 ('very'). Each testing session lasted for around 10 minutes.

\section{4 - Data analysis}

A two-factor multivariate analysis of variance (MANOVA) was conducted. The shape of the cup (tulip, open, or split) and expertise (amateur or expert) were the between-participant factors, and aroma, sweetness, acidity, temperature, and liking were the dependent variables. The variables gender, age, and coffee activity (see Table 1) were also included in the preliminary analysis, but no significant main effects or interactions were revealed, and hence these factors were not included in the main analyses in order to simplify the reporting of results. All basic assumptions of the MANOVA test were checked beforehand and have been fulfilled. All post-hoc comparisons were Bonferroni corrected.

\section{Results}

The between-groups, two-factor MANOVA revealed significant main effects of the shape of the cup $[F(2,275)=9.58, p<0.0001$, Wilks' lambda $=0.72]$ and of expertise $[F(1,275)=2.75$, $p<0.02$, Wilks' lambda $=0.95$ ] on participants' ratings of coffee aroma, taste, and hedonic 
attributes. A significant interaction between these two factors was also observed $[\mathrm{F}(2,275)=4.01, p<0.0001$, Wilks' lambda $=0.87]$.

Post-hoc testing revealed significant effect of the shape of the cup on all of the measured attributes apart from temperature (see Figure 2). In particular, the coffee was rated as more aromatic by the participants who received their sample in the tulip cup $[F(2,275)=8.27$, $p<0.001]$ than by those who received their coffee in the open or split cups. The sweetness $[\mathrm{F}(2,275)=4.66, p<0.01]$ and acidity $[\mathrm{F}(2,275)=5.73, p<0.01]$ ratings given by the participants who tasted the coffee from the split cup were significantly higher than the ratings obtained for either the tulip or open cups [although the difference between sweetness ratings for tulip $(6.10 \pm 0.18)$ and open $(5.83 \pm 0.19)$ shapes approached significance $(\mathrm{p}=0.08)]$. Additionally, those participants who tasted the coffee from the split cup reported liking the coffee significantly less $[\mathrm{F}(2,275)=8.85, p<0.001]$ than those who tasted it from either the tulip or open cups. Post-hoc tests also revealed a significant effect of expertise on liking - with the liking ratings given by the professionals being significantly higher $[\mathrm{F}(1,275)=6.05, p<0.05]$ than those given by the amateurs. That said, no effect of expertise was observed for any other measures (i.e., aroma, sweetness, acidity, or temperature).

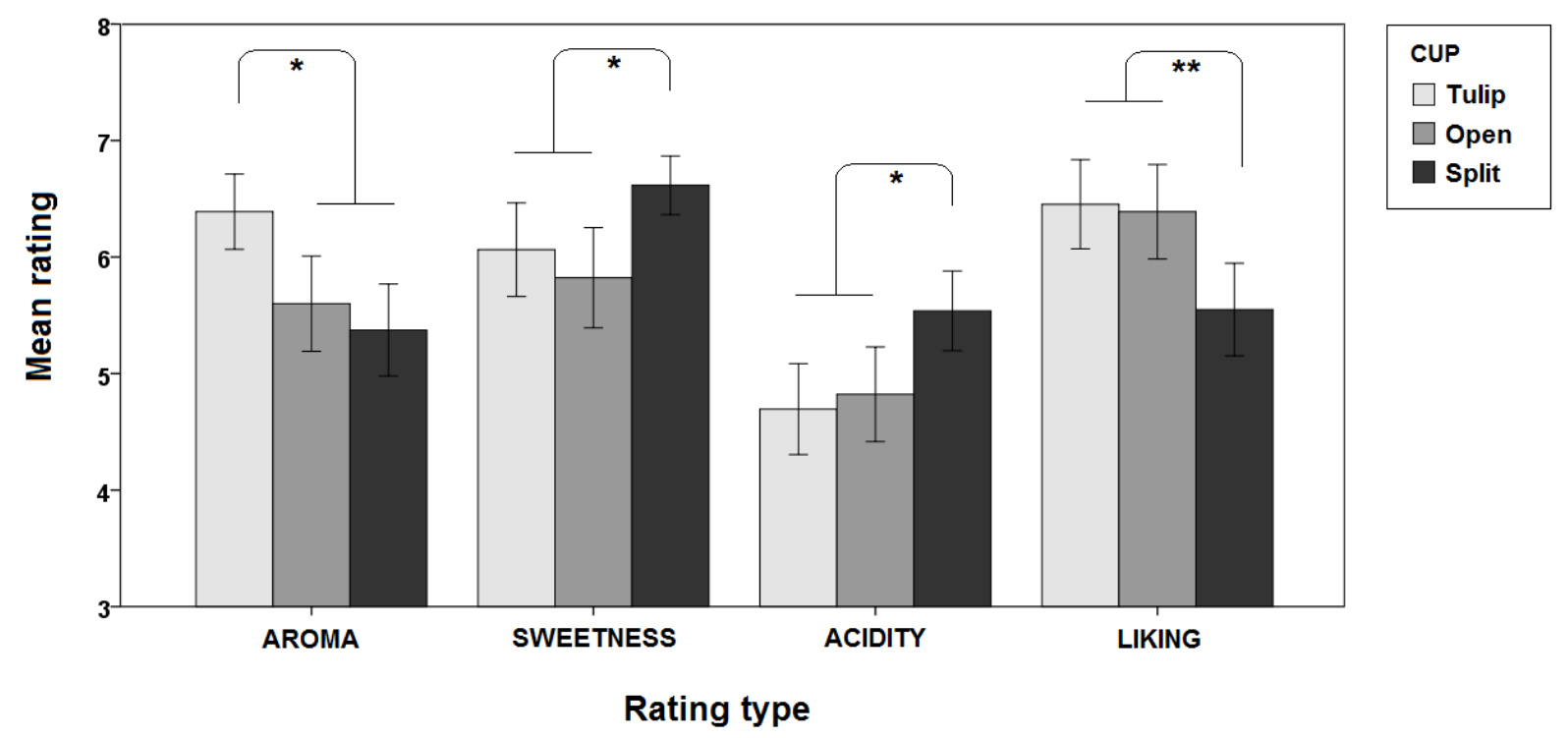

Figure 2: Mean ratings of aroma, sweetness, acidity, and liking, for each of the three cup shapes used in the present experiment (tulip, open, and split). The participants rated the same coffee on a 10-point scale ranging from 0 (not at all) to 10 (very). The error bars highlight the standard errors of the means. Single asterisk ' $*$ ', indicates statistical significance at $\mathrm{p}<.01$, and double asterisks ' $* *$ 'indicate statistical significance at $\mathrm{p}<.001$.

Further pairwise comparisons (using Bonferroni correction) on the significant cup shape by expertise interaction revealed that the amateur participants reported liking the coffee 
significantly less $(4.44 \pm 0.28)$ than the professionals when tasting from the split cup $(6.46 \pm 0.25) \quad(p<0.001)$. The amateur participants also reported liking the coffee significantly more $(6.81 \pm 0.28)$ than the professional participants when tasting from the open cup $(6.04 \pm 0.26)(p<0.05)$. Furthermore, the amateur participants rated the aroma of the coffee from the split cup as significantly less intense $(4.95 \pm 0.28)$ than did the professional participants $(5.72 \pm 0.25)(p<0.05)($ see Figure 3$)$.
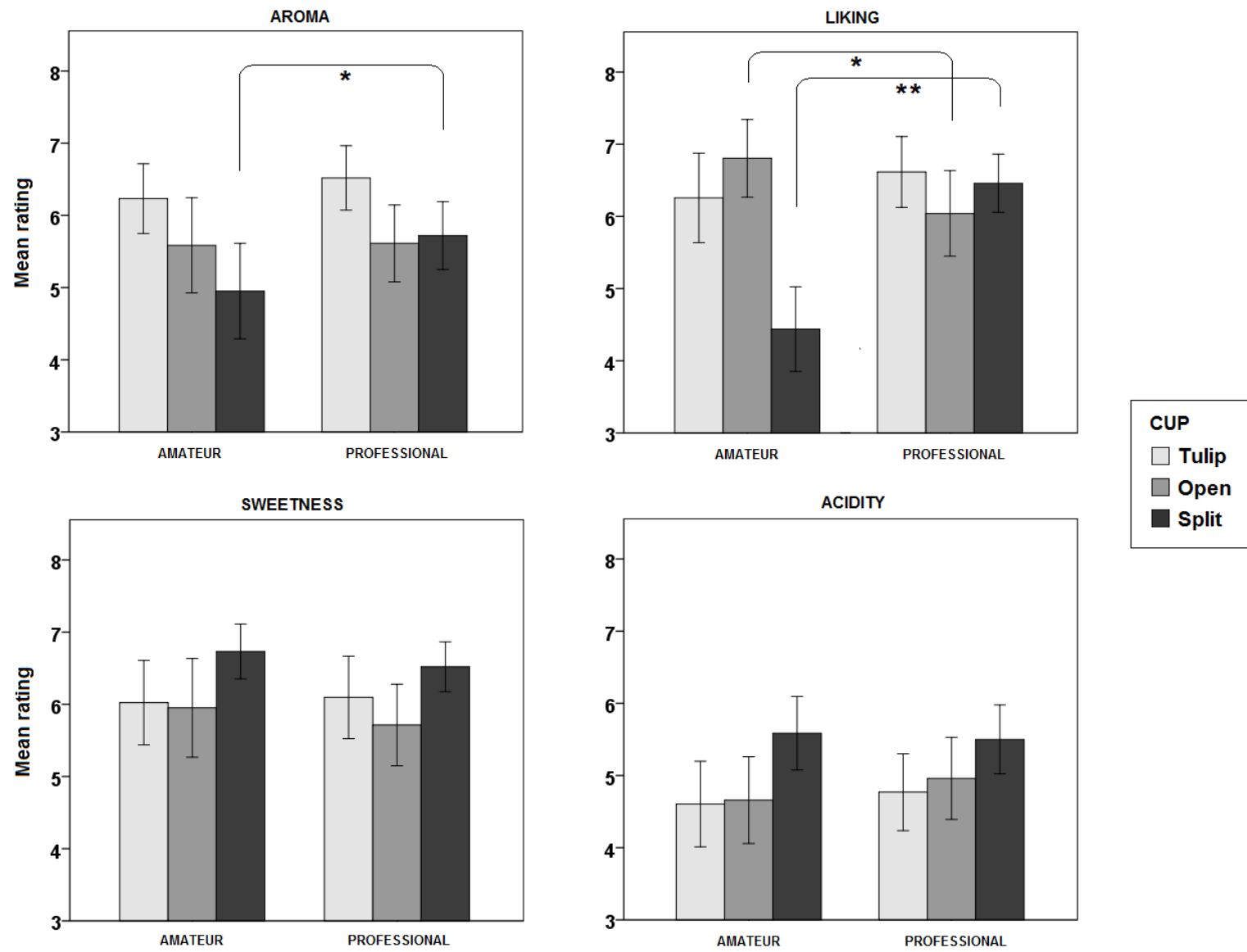

Figure 3: Mean ratings of aroma, liking, sweetness, and acidity for each of the three cup shapes used in the experiment (tulip, open, and split), as a function of the level of expertise of the participants (amateur vs. professional). The error bars show the standard errors of the means. '*' $\mathrm{p}<.05$, '**' $\mathrm{p}<.0001$.

\section{Discussion}

In the present study, we tested whether three different shapes of coffee cup (namely tulip, open, and split) would exert any kind of influence on the ratings of aroma, flavour attributes as sweetness and acidity, and liking given by specialty coffee professionals and amateurs. The results of this large-sampling experiment corroborate the general claim that the consumers' evaluation of aroma, flavour, and hedonic value are influenced by the shape of the coffee cup, as has been shown to be the case for wine (e.g., Venturi et al., 2016). One 
fundamental dichotomy to be considered with respect to the interpretation of the results is how physical versus psychological variables concerning the shape of the cup may have had an effect on aroma and flavour perception judgements. Specifically, the present data highlight the fact that the shape of the cup impacts he perception of coffee aroma. It is well-known that the size and shape of the wine glass influences the release, and/or the retention, of the wine volatiles, as well as the oxygenation level over time (Parpinello et al., 2018). According to the wine literature, there is a positive correlation between the perceived aroma intensity and the ratio of maximum diameter to opening diameter (D-ratio) (see Spence \& Wan, 2015 for a review). Such a result undoubtedly makes sense, since a larger diameter would allow the volatilization of odour compounds which would then be retained within the glass due to the narrow opening (e.g., Fischer \& Loewe-Stanienda, 1999). Given the number of volatiles compounds that have been documented in coffee (Yeretzian, 2017), one might expect that the physical properties of the cup may have an effect on the perception of complex aromas in specialty coffee as well. Indeed, the aroma of the coffee was rated as more intense by both amateurs and professionals when evaluated from the tulip-shaped cup. In the present study, the tulip cup is the one with the highest 'D-ratio', which indicates that the physical properties of the receptacle do indeed play a role when it comes to the perception of complex odours, in both wine and specialty coffee. Furthermore, the professionals rated the aroma intensity in the split cup higher than did the amateurs. The split cup has a bulbous shape below the narrowing indentation designed to retain the volatiles. Its bulbous bottom resembles a short tulip cup, having a positive 'D-ratio' if the diameter of the indentation is considered as the opening diameter. However, any increased retention of steam seems to have only a subtle effect on aroma, since only the trained experts were able to detect it - and even so not differently from the open shape cup.

Psychological aspects on the shape affecting the perception of aroma should also be considered here. Human-product interaction is part of a multisensory experience which also includes linguistic or symbolic conceptualizations of the product's/object's appearance and afforded actions (Abrahamson \& Lindgren, 2014). For instance, van Rompay et al. (2005) have demonstrated that objects (e.g., a jar) presenting high levels of closure may connote meanings implying a sense of constrictiveness and security. In the present study, in addition to the physical effect, it is possible that the participants might have associated enhanced closure of form with greater constrainedly of contents, leading to greater intensity of the perceived odour. 
It is interesting to note that the two taste attributes assessed - i.e., sweetness and acidity were given significantly higher ratings by both amateur and professional participants when tasting the coffee from the split cup. Regarding sweetness, several studies have demonstrated a crossmodal correspondence between basic tastes and angular-to-round shapes (see Spence \& Ngo, 2012 for a review). The strongest and most consistent of these correspondences concerns the matching of sweet tastes with round shapes (Spence, 2012; Velasco et al., 2016). Visually-curved shapes (when compared to angular shapes), have been shown to increase a taster's sensitivity to sweetness even at threshold levels (Liang et al., 2013). Rounded tactile patterns on the outer surface of the cup have also been shown to increase the perceived sweetness of hot chocolate and brewed coffee when compared to more angular surface patterns (see Van Rompay et al., 2017). In fact, round shapes have been shown to influence both expectation and the actual perception of sweetness in different foods and drinks (see Velasco et al., 2016, for a review). Visual product cues indicate what the consumer is about to experience by putting her mind in an anticipatory, or predictive, mode that can be powerful enough to modify the pattern of activation seen in primary sensory regions. For instance, neuroimaging data shows that the expectation of sweetness primed by a 'very sweet' label increased the perceived sweetness of the orange juice as well as the anterior insula activation (region which has been identified as primary taste cortex based on previous taste-related studies) (Woods et al., 2011). Taste expectations were not measured in the present study; Nevertheless, given the well-investigated crossmodal association between sweetness and roundness, it is justifiable to suggest that the increased perceived sweetness could have resulted from a multisensory process, given that the tasters had direct contact with the cup they could both see and touch its roundness (cf. Obrist et al., 2014; Van Rompay et al., 2017). If fact, the sweetness ratings for the tulip cup were borderline-significantly higher than those for the open cup. Despite the shape of the three cups could be considered as 'round', both the visual and tactile aspects of their shapes deliver a quite clear gradation of roundness. This may imply an ascending gradation of perceived sweetness as a function of the roundness of the cup (open $<$ tulip $<$ split).

As yet, ratings of acidity were highest when the coffee was tasted from the split cup. The mechanism behind this finding is not as clear-cut as that supporting the results concerning aroma and sweetness. First, we have to consider previous suggestions from the wine literature that glasses with different shapes may interfere with oral sensation as a result of a particular 
flow pattern of the wine across the taster's tongue (e.g., Grainger, 2009; Peynaud, 1987). These views account for either the difference between narrow and wide opening glasses, or the difference between fine and beaded rims. They assume that the physical shape of the glass helps direct the liquid to particular locations on the surface of the tongue, and that this variation in flow patterns would lead to differences in the perception of specific taste compounds. However, detailed investigations that could provide convincing evidence to support such an explanation are still missing. For instance, Hummel et al. (2003) tested four different shapes of wine glasses and found that acidity was rated as most intense in the intermediate opening diameter glass - also, all the glasses used had fine rims. This hypothesis cannot explain our results either. It is not clear why the split cup, which has the same opening diameter as the open cup, would increase the perception of acidity whilst the open cup would fail to do so. In addition, all cups used had the same thickness of rim.

This finding of increased acidity (together with increased sweetness) in the split cup could be approached in terms of the 'supra-additive' effect observed in odour-taste crossmodal associations. For instance, Lim et al. (2014) investigated retronasal odour enhancement by means of taste, and found that only sucrose - compared to citric acid and caffeine significantly enhanced the perceived intensities of retronasal citrus odour. From this, a possible interpretation for our results might be that the perception of sweetness (increased by the round-sweet crossmodal correspondence discussed above) could have enhanced perceived intensities of the citrus retronasal odour attribute of the coffee in a bottom-up manner. Furthermore, Sunarharum (2016) assessed flavour attributes in different specialty coffees and found a strong positive correlation between the 'citrus' and 'fruity' attributes, which, in turn, showed a positive correlation to 'sourness'. Based on such an observation, it could be speculated that once the unitary sweet-citrus flavour of the coffee was perceived, it might have elicited 'acidity' in a top-down fashion - in a kind of crossmodal categorisation that does not require explicit conceptual identification (Spence \& Deroy, 2013). In future research on drinking receptacles, it would therefore perhaps be worth including measurements of those flavour attributes often present in specialty coffee (e.g., fruity, floral, nutty).

As we have not directly tested the halo effect as a contributor of odour-induced taste enhancement in the present experiment, it cannot be ruled out. However, given that both tastes assessed were enhanced in the split cup, which was the cup with the lowest hedonic 
and aroma ratings, we believe that the potential contribution of halo effects was probably minor.

Overall, both amateurs' and experts' ratings of aroma and taste were significantly influenced by the shape of the cup in which the coffee happens to be served. These observations are in agreement with previous research in wine demonstrating that the glass shape and dimensions affect aroma and flavour judgements of social drinkers and professional wine tasters alike (see Spence \& Wan, 2015, for a review). It is interesting to note that the coffee tasted from the split cup received the lowest liking scores. The effect of expertise observed in these liking scores is also worth noting, given that the amateurs gave significantly lower ratings than those given by professionals. Assuming that enhanced sweetness and acidity perceived in the split cup is not related to the unpleasantness of the coffee itself (given the professionals' ratings), a plausible explanation for this is that the split cup might have been seen as an unusual drinking receptacle for coffee. With no previous exposure to that particular shape, the split cup would not match any of the participants' everyday drinking experience, including the experience of drinking coffee. Prior research has shown that consumers tend to prefer more typical designs because of the ease with which a typical design can be processed - design typicality is a stimulus-inherent determinant of perceptual fluency, with high levels of novelty leading to lower pleasure (Landwehr et al., 2013; Winkielman et al., 2006). Despite having not directly evaluated the aesthetic response of the participants to the split cup, the lower liking ratings given by the amateurs may perhaps indicate lower processing fluency due to the high atypicality or complexity of the drinking receptacle itself. On the other hand, professional participants are used to grading coffee from cupping vessels that come in varying shapes, and are trained to try and attend not to the vessel, but to the sensory attributes of the coffee itself (inasfar as this is possible).

Two additional factors that could have influenced the results obtained in the present study should also be taken into account, namely (1) the cultural background of the population tested, and (2) the specific sensory attributes of the coffee used as a sample. Many crosscultural studies have shown significant differences in taste, flavour, and hedonic judgements of several beverages in relation to visual cues such as colour of the drink (Shankar et al., 2010) and even the shape/type of receptacle (Wan et al, 2015; Van Doorn et al., 2017), although some studies have reported invariances in cross-cultural crossmodal correspondences as well (Liang et al., 2016; Spence et al., 2015). Concerning the sensory 
profile of the sample, some authors - including wine experts - have claimed that matching specific glassware to the style of wine (or grape variety) can enhance the drinking experience (Cloake, 2012; Venturi et al., 2016), although it is not clear whether the differences in aroma and flavour perceived by wine experts would necessarily always be appreciated by the amateur drinkers (Spence, 2011; Spence \& Wan, 2015). Due to the lack of empirical studies to date concerning how the receptacle affects sensory and hedonic judgments in specialty coffee, further research is needed in order to elucidate how visual and/or tactile aspects of the receptacle would affect the consumer's experience in different populations (e.g., cultural background, expertise) and in different coffee sensory profiles (e.g., terroir, varietal).

\section{Conclusion}

In summary, the results of the present study demonstrate the profound impact that the shape of the coffee cup has on both flavour and hedonic judgments in both amateur and expert specialty coffee consumers. All the assessed attributes were affected by the shape of the cup. The effects observed on the attributes 'aroma' and 'sweetness' corroborate commonly reported findings on the association between bulbous-shaped glasses and aroma intensity, and the correspondence between round shapes and sweet tastes. On the other hand, the effect of the shape of the cup on perceived acidity is not so clear in light of previous research on crossmodal correspondence.

Given that the drinking receptacle is a necessary part of the multisensory coffee drinking experience, it is crucial to realize just how much of an influence the receptacle actually has on the consumer's perception of the contents. Ultimately, drinking receptacles should most likely be carefully designed in order to optimize the consumer's experience of the contents (e.g., Stead et al., 2014), and/or to match a given consumer's particular taste world/preferences.

\section{Acknowledgements}

The first author was privileged to have "The Coffee Sensorium" project embraced and assisted by several people from the specialty coffee community. This project owes a great deal to Tim Wendelboe, Mariana Proença (from the Brazilian International Coffee Week), Diogo Dias (from Recreio Estate Coffee), Camila Arcanjo (from Sindicafé - SP), and Bourbon Specialty Coffees (for the training provided on specialty coffee cupping methods). 


\section{References}

Barbosa, J. N., Borém, F. M., Cirillo, M. Â., Malta, M. R., Alvarenga, A. A., \& Alves, H. M. R. (2012). Coffee quality and its interactions with environmental factors in Minas Gerais, Brazil. Journal of Agricultural Science, 4(5), 181.

Benzi, F. (2008). The flavourist. In H. Blumenthal (Ed.), The big Fat Duck cookbook (pp. 476-478). London, UK: Bloomsbury.

Bury, C. (2014). Does the cup influence the taste of the coffee? http://coffeestrides.blogspot.com.br/2014/03/does-cup-influence-taste-of-coffee.html (accessed 27.01.18).

Cavazzana, A., Larsson, M., Hoffmann, E., Hummel, T., \& Haehner, A. (2017). The vessel's shape influences the smell and taste of cola. Food Quality and Preference, 59, 8-13.

Cloake, F. (2012). Can the shape of your glass enhance the taste of the wine? Do you need to change your glass depending on what you're drinking? http://www.theguardian.com/lifeandstyle/2012/apr/25/shape-of-glass-enhance-wine (accessed 18.01.18).

Delwiche, J. F. (2004). The impact of perceptual interactions on perceived flavor. Food Quality and Preference, 15, 137-146.

Donnet, M. L., Weatherspoon, D. D., \& Hoehn, J. P. (2007). What adds value in specialty coffee? Managerial implications from hedonic price analysis of Central and South American e-auctions. International Food and Agribusiness Management Review, 10, 1-18.

Fischer, U., \& Loewe-Stanienda, B. (1999). Impact of wine glasses for sensory evaluation. International Journal of Vine and Wine Sciences, Wine Tasting, Special Edition, 33 (Suppl. 1), $71-80$.

Gatti, E., Spence, C., \& Bordegoni, M. (2014). Investigating the influence of colour, weight, $\&$ fragrance intensity on the perception of liquid bath soap. Food Quality \& Preference, 31, $56-64$.

Grainger, K. (2009). Wine quality: Tasting and selection. Chichester, UK: John Wiley \& Sons.

Kampfer, K., Leischnig, A., Ivens, B. S., \& Spence, C. (2017). Touch-taste-transference: Assessing the effect of the weight of product packaging on flavor perception and taste evaluation. PLoS ONE, 12(10).

Laing, D., \& Francis, G. (1989). The capacity of humans to identify odors in mixtures. Physiology \& Behavior, 46, 809-814. 
Laing, D. G., Link, C., Jinks, A. L., \& Hutchinson, I. (2002). The limited capacity of humans to identify the components of taste mixtures and taste-odour mixtures. Perception, 31, 617635.

Landwehr, J. R., Wentzel, D., \& Herrmann, A. (2013) Product design for the long run: Consumer responses to typical and atypical designs at different stages of exposure. Journal of Marketing, 77(5), 92-107.

Liang, P., Biswas, P., Vinnakota, S., Fu, L., Chen, M., Quan, Y., Zhan, Y., Zhang, G., \& Roy, S. (2016). Invariant effect of vision on taste across two Asian cultures: India and China. Journal of Sensory Studies, 31(5), 416-422.

Liang, P., Roy, S., Chen, M.-L., \& Zhang, G.-H. (2013). Visual influence of shapes and semantic familiarity on human sweet sensitivity. Behavioural Brain Research, 253, 42-47.

Lingle, T. R., \& Menon, S. N. (2017). Cupping and grading-Discovering character and quality. In B. Folmer (Ed.), The craft and science of coffee (pp. 181-203). London, UK: Academic Press.

Lynch, J. G., \& Zauberman, G. (2007). Construing consumer decision making. Journal of Consumer Psychology, 17(2), 107-112.

Mirabito, A., Oliphant, M., Van Doorn, G., Watson, S., \& Spence, C. (2017). Glass shape affects the perceived taste of beer. Food Quality and Preference, 62, 257-261.

Obrist, M., Comber, R., Subramanian, S., Piqueras-Fiszman, B., Velasco, C., \& Spence, C. (2014). Temporal, affective, and embodied characteristics of taste experiences. In Proceedings of the 32nd annual ACM conference on Human factors in computing systems CHI '14 (pp. 2853-2862). New York, New York, USA: ACM Press.

Okamoto, M., \& Dan, I. (2013). Extrinsic information influences taste and flavour perception: A review from psychological and neuroimaging perspectives. Seminars in Cell \& Developmental Biology, 24(3), 247-255.

Parpinello, G. P., Meglioli, M., Ricci, A., \& Versari, A. (2018). Effect of different glass shapes and size on the time course of dissolved oxygen in wines during simulated tasting. Beverages, 4(1):3.

Peynaud, E. (1987). The taste of wine: The art and science of wine appreciation (Trans. M. Schuster). London, UK: Macdonald \& Co.

Piqueras-Fiszman, B., \& Spence, C. (2015). Sensory expectations based on product-extrinsic food cues: An interdisciplinary review of the empirical evidence and theoretical accounts. Food Quality \& Preference, 40, 165-179.

Rapp, A. (1990). Natural flavours of wine: Correlation between instrumental analysis and sensory perception. Fresenius' Journal of Analytical Chemistry, 337, 777-785.

Rhinehart, R. (2009). What is specialty coffee? http://www.scaa.org/?page=RicArtp1 (accessed 18.01.18). 
SCA (2015) http://www.scaa.org/PDF/resources/cupping-protocols.pdf (accessed 18.01.18).

SCA (2017a) http://www.scanews.coffee/2017/11/29/2017-u-s-specialty-coffeeconsumption-trends/ (accessed 18.01.18).

SCA (2017b) http://www.scanews.coffee/2017/11/28/2017-western-european-coffee-marketsize-report/ (accessed 18.01.18).

Schwan, R. F., Silva, C. F., \& Batista, L. R. (2012). Coffee fermentation. In Y. H. Hui (Ed.), Handbook of plant-based fermented food and beverage technology (pp. 677-690). Boca Raton, FL: CRC Press.

Shankar, M. U., Levitan, C., \& Spence, C. (2010). Grape expectations: The role of cognitive influences in color-flavor interactions. Consciousness \& Cognition, 19, 380-390.

Small, D. M. (2012). Flavor is in the brain. Physiology \& Behavior, 107, 540-552.

Spence, C. (2011). Crystal clear or gobbletigook? The World of Fine Wine, 33, 96-101.

Spence, C. (2015). Multisensory flavour perception. Cell, 161, 24-35.

Spence, C., \& Deroy, O. (2013). Crossmodal mental imagery. In S. Lacey \& R. Lawson (Eds.), Multisensory imagery: Theory and applications (pp. 157-183). New York, NY: Springer.

Spence, C., \& Ngo, M. (2012). Assessing the shape symbolism of the taste, flavour, and texture of foods and beverages. Flavour, 1:12.

Spence, C., \& Piqueras-Fiszman, B. (2014). The perfect meal: The multisensory science of food and dining. Oxford, UK: Wiley-Blackwell.

Spence, C., \& Van Doorn, G. (2017). Assessing the impact of the shape of the drinking receptacle on taste/flavour perception: A review. Beverages, 3:33.

Spence, C., \& Wan, X. (2015). Beverage perception \& consumption: The influence of the container on the perception of the contents. Food Quality \& Preference, 39, 131-140.

Spence, C., \& Wang, Q. (J.) (submitted). On the meaning(s) of complexity in the chemical senses. Chemical Senses.

Spence, C., Wan, X., Woods, A., Velasco, C., Deng, J., Youssef, J., \& Deroy, O. (2015). On tasty colours and colourful tastes? Assessing, explaining, and utilizing crossmodal correspondences between colours and basic tastes. Flavour, 4(1), 23.

Styger, G., Prior, B., \& Bauer, F. F. (2011). Wine flavor and aroma. Journal of Industrial Microbiology \& Biotechnology, 38(9), 1145.

Sunarharum, W. B. (2016). The compositional basis of coffee flavour. $\mathrm{PhD}$ Thesis, Queensland Alliance for Agriculture and Food Innovation, The University of Queensland. doi:10.14264/uq1.2016.1110 
Teuber, R., \& Herrmann, R. (2012). Towards a differentiated modeling of origin effects in hedonic analysis: An application to auction prices of specialty coffee. Food Policy, 37, 732740 .

Van Doorn, G., Woods, A., Levitan, C. A., Wan, X., Velasco, C., Bernal-Torres, C., \& Spence, C. (2017). Does the shape of a cup influence coffee taste expectations? A crosscultural, online study. Food Quality and Preference, 56, 201-211.

Van Rompay, T. J., Finger, F., Saakes, D., \& Fenko, A. (2017) "See me, feel me": Effects of 3D-printed surface patterns on beverage evaluation. Food Quality \& Preference, 62, 332-339.

Velasco, C., Woods, A. T., Petit, O., Cheok, A. D., \& Spence, C. (2016). Crossmodal correspondences between taste and shape, and their implications for product packaging: A review. Food Quality \& Preference, 52, 17-26.

Venturi, F., Andrich, G., Sanmartin, C., Taglieri, I., Scalabrelli, G., Ferroni, G., \& Zinnai, A. (2016). Glass and wine: A good example of the deep relationship between drinkware and beverage. Journal of Wine Research, 27, 153-171.

Verhagen, J. V., \& Engelen, L. (2006). The neurocognitive bases of human multimodal food perception: Sensory integration. Neuroscience and Biobehavioral Reviews, 30, 613-650.

Wan, X., Zhou, X., Woods, A. T., \& Spence, C. (2015). Influence of the glassware on the perception of alcoholic drinks. Food Quality and Preference, 44, 101-110.

Winkielman, P., Halberstadt, J., Fazendeiro, T., \& Catty, S. (2006). Prototypes are attractive because they are easy on the mind. Psychological Science, 17, 799-806.

Woods, A. T., Lloyd, D. M., Kuenzel, J., Poliakoff, E., Dijksterhuis, G. B., \& Thomas, A. (2011). Expected taste intensity affects response to sweet drinks in primary taste cortex. Neuroreport, 22, 365-369.

Xu, J. (2003). Coffee. Journal of Agricultural \& Food Information, 5(3), 79-86.

Yeretzian, C. (2017). Coffee. In A. Buettner (Ed.), Springer handbook of odor (pp. 107-128). Cham, Switzerland: Springer International Publishing. 